\title{
The interaction design research tourism APP application under UGC mode
}

\author{
Xue Bai ${ }^{1}$, Xiaozhang Zhang ${ }^{1}$ \\ ${ }^{1}$ School of Art and Design,Wuhan University of Science and Technology, China
}

Keywords: UGC mode, travel APP, application, interaction design

\begin{abstract}
The UGC model widely attention in the field of mobile Internet tourism. How to better grasp the UGC mode user requirements, constantly optimize the user experience, conceived the interactive process more fluent and excellent visual interface effect, so as to get more users, has become a tourist class APP developer to issues of common concern. This article through to the UGC mode travel APP user research, in-depth analysis of user behavior and demand, from the information architecture, task, process design, navigation design, and detail design, preliminary summarized UGC mode travel APP interaction design principles.
\end{abstract}

\section{Introduction}

Some tourists believe that travel can broaden horizons, increase knowledge, they are willing to record and share at the end of the journey on the road, see, smell, want some positive travel enthusiasts in some factors under the influence of has a strong desire to share the experience. With the popularity of social networking sites and mobile phone APP, tourists will be at the end of the journey and the journey to share travel information anytime and anywhere [1]. Currently, give priority to with user-generated content of tourism of social networking sites and mobile terminal APP, such as "day of cellular free line" travel, bread, etc. With its rich UGC content and a large number of users in the online travel market occupied a place.

\section{The present situation of the UGC mode travel APP}

UGC (User Generated Content) denote "published on the Internet in any way by the User creation of text, images, audio, video and other Content", from the earliest web application and popularization of network technology. The world organization for economic cooperation and development in a 2007 report describes the UGC three characteristics [1]:

1) On the premise of network publishing;

2) Content with a certain degree of innovation;

3) Non-specialists or authority organization. Common types of UGC with social networking, video sharing, photo sharing, knowledge sharing, and community BBS and micro blog, etc. Travel APP UGC generally refers to the user through the APP to share their travel, all kinds of tourism information and tourism strategy, form mainly released photos, text, video, etc.

The rapid development of the intelligent terminal and mobile network make the traditional tourism and convergence speed of the mobile Internet. Users through various travels APP can always grasp the latest tourism information, real-time booking air tickets, hotel, booking tickets, etc. Mobile travel has become the keywords of the tourism industry, various types of tourism APP has also become the mobile phone users installed for the application.

\section{The user research of UGC mode travel APP}

User behavior research. The author after the depth interview was carried out on the respondents came to the conclusion [2]:

a. UGC model tourism, the active users of the APP has formed the habit of sharing. In a variety of internal motivation and external factors, they are very willing to give their travel experience and 
contribution to other users. For active users, they more need to be able to help them to show themselves, realize self-worth.

b. UGC mode the enthusiasm of the travel APP ordinary users to contribute content are less active users, but such user content also want your contributions can be recognized more and more people. In the process of travel and will let them share their trip photos get satisfaction.

c. have experiences of UGC mode travel APP users are not good at screening from a large amount of information.

User demand research. User demand for products can be divided into functional requirements and experience requirements. Function demand refers to the user needs a function to help its implementation by the product purpose: experience demand refers to the user in the process of using product to product visual effects in terms of ease of use and interface requirements [2].

a. The functional requirements. Active users UGC mode travel APP has formed the habit of sharing. In a variety of internal motivation and external factors, they are very willing to give their travel experience and contribution to other users. For active users, they more need to be able to help them to show themselves, realize self-worth. UGC mode the enthusiasm of the travel APP ordinary users to contribute content are less active users, but such user content also want your contributions can be recognized more and more people. In the process of travel and will let them share their trip photos get satisfaction.

b. Experience requirements. In addition to the functional requirements, user experience quality directly affects the user use the enthusiasm. Used according to the UGC travel APP users of the results of questionnaire survey, the "rich in content, practical, reliable information" and "smooth operation, simple operation steps," the most valued by users. UGC mode active users travel APP for product the interface of visual effect and interaction experience have more requirements [1]. Interface is simple, visual effects, good product can stimulating the enthusiasm of them contribute more. UGC mode travel APP normal user content's enthusiasm is often restricted to the contribution of the complex task process, such as "write travelogue" function. Such user wants to publish content more simple operation process, reduce the time of the study and explore. Have experiences of UGC mode travel APP users are willing to share their travel experiences, but they are not familiar with the UGC mode of travel APP, if recommended they use, they will pay more attention to the task of operation process is easy to learn, they will lose interest if too complicated.

User exists in the whole process of tourism travel information demand, at the end of the travel and travel also has a record of sharing and travel demand. Both can be mutual transformation, the contents of the UGC mode travel APP and is most of the information from the user's share. Therefore, the incentive for content sharing and contribution is necessary. In addition to external rewards, also should pay attention to user generated content with the interaction between product interface, and optimize the task process, improve the user experience.

\section{The interface interaction design travel APP under UGC mode}

Compared with other types of travel APP, the greatest characteristic of UGC mode travel APP is most of its contents and information from the user's share, user generated content in the task of process and the product will produce many complex interactions between interfaces. Product information architecture logic is clear, the task process design is reasonable, the user can complete the task quickly, easily and smoothly, interface can give users a timely feedback, these factors can directly affect the user participation motivation [3]. Good interaction process can help users better contribute to complete the task, motivate users to produce more high quality content. Therefore, research on this kind of product of interface interaction design is very important to improve product user experience.

UGC mode travel APP interface interaction design should pay attention to the following [3]:

1) The UGC model travel APP content is very rich, the designer needs to design better information architecture to organize the content, so as to help users better access to information; 
2) The UGC model tourism function of user generated content in the APP, such as "to write travel notes", "write comments on" function should be to optimize task process, user generated excitation by the better operation experience more high quality content;

3) The UGC mode should be paid attention to travel APP detail design of the interface, the user guide and feedback in time, help users to finish operation task better.

Information Architecture. It is the body of the object information, is decided by information architects to design structure, organization and classification, let users and users to easily find and manage a science and art of information architecture design is between information and users to establish a channel, enabling users to access to the information you want. Effective information architecture can according to the actual needs of the user to complete the task step by step to guide the user to get the information they need [3].

"Day of cellular free line" APP USES the information architecture is the hierarchical structure with natural structure. The core value of the products is to provide customers with all kinds of tourism information, and information quantity is very large, so they must be good will organize all the information presented to the user.

Information architecture of mobile phone APP is not single, but in combination with each other. Such as natural structure is generally don't separate use in the product information architecture design, general combined with hierarchical structure. "Day of cellular free line" of "book hotel" features, for example, some users and no clear goals, they choose the function of the entrance into the hotel reservation directly from the home page. Some users have had clear objectives, their search in the destination before you enter the destination hotel list page.

User stories can be divided into two modes: task and browsing. The characteristics of the task type are the user to complete the task, for the purpose of accurate and efficient, for example destination hotel information queries [4]. Browse type is characterized by the user without purpose, fragmentation, enough time, casual, such as brush Weibo aimlessly. "Look travel notes", for example, some users do not have clear goals, use the fragment time to look at other users write travelogue, choose direct access to the home page of "travel" or "bee first travel notes"; But some users have clear goals, such as there has been a want to go to tourist destination, after entering "travel", search the travel destination. Or directly through search destination, into the destination page "travel" and then enter the destination. Two ways can let users won the product content and information, to give attention to two or more things "task type" and "browse type" two users use scenarios. This design is also the embodiment of the natural combination of structure and hierarchy.

Natural structure and hierarchy combination of may let users in the first contact with the product there is a learning process [4]. Therefore, "day of cellular free line" APP needs to be improved: as much as possible to optimize task process, allows users to quickly locate you need content;Add new guidance, let the new user to quickly understand the product function, reduce the time to study. UGC mode travel APP information architecture design should not only choose a model, but to fully according to users demand to the function, behavior characteristics and flexible use of operating habits.

The process design of the task. Task process design refers to the users to perform a series of actions to achieve a certain goal or for the analysis of the cognitive process of an action. By task analysis, the designer can clearly understand the current APP is going to realize the function of the hierarchical order and the information transfer in the system of the state, in the structure of the design can grasp the global product and the user is currently required for operation before the design process must first learn the user's goal, then to the operation of the user to achieve the goal of dismantling, at this time every operation visual as a primary task [4]. On the basis of the original task, according to the level of demand is the task in more detail into several subtasks. UGC mode travel APP in typical UGC function "to write travel notes", for example, "to write travel notes" the task of process is more complex. The author of Tao in the road "to write travel notes" function of community in-depth experience after the draw out the flow chart of its task. According to the flow chart of tasks can be seen, for the whole task "to write travel notes" can be broken down into "add notes", "add photos", "add video" the three major subtasks, the subtasks can split again, again, for example, "modified 
shooting time for adding good pictures". The complex task of process very test the user's ability to learn so must be optimized and streamlined.

In addition, the design should also consider the user's task flow model, such as user when doing a habitual thinking and logic. After research the user found that users shared their travel experiences since the end of the travel demand [5]. After the author's observation, the user in the process of writing travel is often done through the order of the memories, such as the first day to arrive after went to where to play, to eat what food, entertainment, in which the hotel accommodation and so on, the user according to the order of memory to upload the corresponding travel photos and description for each item. "To write travel notes" in the design the function of task process should not violate the user when the chariot, principle model, disrupt this also, mental model will allow users to create chaos, lose confidence to finish operation task.

Navigation design. Navigation of mobile phone APP and realistic signpost or map works in a similar way. It is mobile phone APP virtual framework, to the user with instructions and identify the role of. Navigation of mobile phone APP user experience plays a key role. At present there are mainly eight mobile APP navigation design pattern: tag navigation, rudder drawer type navigation, navigation, list of navigation, navigation, navigation TAB navigation [6].

UGC mode travel APP navigation design must highlight the product of nuclear function point, try to be flat task path. Clear navigation and location information can effectively avoid users get lost in the operation. "Day of cellular free line" of the primary navigation is simpler, the hierarchy number is 3 layers, but is very wide, each level contains the contents of the user easy to get lost in the operation, only by constantly return to determine their own position.

Bread travel navigation USES a class type, outstanding new long travel and life story of the entrance [5]. Old user opens the APP can record their own travel and story;New users can learn about sending a speed function;If entrance hidden too deep, you will be hard to attract the attention of the new user.

Design detail. UGC mode travel APP navigation design principle is must be able to let users through rapid positioning navigation to the position of the content they want. To comb through the navigation, the content of the confused, as far as possible to reduce the user study time of the process for each task [6].

a. Feedback prompts design. Feedback tip is to let the user know what the next step of operation is. Friendly feedback or no feedback will be helpless or displeasure of negative feedback to the user experience. Prompt feedback hint of a lot of more pyretic, any feedback should be properly opportunely time appear in the right place.

UGC mode travel APP feedback design principles. At the different stages of the operation task for the user to provide necessary feedback, positive, instant feedback, especially in writing travel notes and write comments on the task in the process, feedback tip should accompany the whole process of the user action. Reasonable design feedback tip number, try not to disturb the user operation. Give the user feedback information must allow the user to use the most convenient way to complete. Don't interrupt the user's way of thinking, to avoid keep out users may view or operation of the object, let the user operate more smoothly.

b. Guide the design. Mobile APP to guide design enables the user to quickly familiar with the product, in front of the user in difficulty to give timely help. Strive to guide design simple and interesting, at the right time to appear in the right place [6]. UGC travel APP guide design is very important; it can let users faster familiar with the process of application of a variety of tasks, to reduce the time to study. Especially in the "to write travel notes" task in the process of the need for various forms of guide design, help the user to complete all operations.

c. Animation design. Animation design can make the phone APP experience more dynamic, more emotional [5]. Mobile phone APP animation design includes dynamic switching between pages, feedback page loading animation and animation, etc. UGC mode travel APP interface interaction design should give full play to the role of the animation, give product emotional characteristics. Beautiful animation effects can make the user feel shine at the moment, giving users more pleasant user experience. 


\section{Conclusion}

Tourism APP market competition is fierce, the user's choice of travel APP also gradually increasing. The existing mode of UGC travel APP on the market of the powerful and rich in content, but still need to improve and optimize the interface interaction design. UGC mode of travel APP want to win the user's favor, in addition to the innovation of the content of the control and function, should pay more attention to the interaction of the interface design, and constantly optimize the user experience, so as to increase the competitiveness of the products.

\section{Acknowledgement}

Article from Project: Research projects of Humanities and social science Hubei province.Project name: Application research of Hubei Jingchu traditional patterns and cultural connotation in the tourism product development, Project item number: 13Q021.

\section{References}

[1] Y.X.Zhao, User generated content (UGC) concept analysis and the research progress,Journal of Chinese library, 2012, vol.5, pp.68-71.

[2] X.T. Wu, Product system design, China light industry press, 2002, vol. 5, pp. 21-24.

[3] Sh.L. Liu,Humanistic care of modern product industrial design, Fashion design, 2001, vol. 7, pp. 8-11.

[4] K.Q. Li, Analyses the modern products humanized design, Engineering machinery Chang'an University, 2005, vol. 5, pp. 2-6.

[5] S.T. Sun, The analysis and discussion the era of wireless network travel APP, Journal of Hubei economy academy, 2013, vol. 10, pp. 49-52.

[6] X.F. Xiao, Mobile design, The electronic industrial press, 2013, vol. 7, pp. 17-21. 